\title{
A low complexity peak-to-average power ratio reduction scheme using gray codes
}

\begin{abstract}
A low-complexity peak-to-average power ratio (PAPR) reduction scheme in an orthogonal frequency division multiplexing system is proposed. The proposed scheme utilizes a new phase sequence based on a gray code structure and a similarity measurement block. Due to the ordered phase sequences, a noteworthy reduction capacity is obtained in terms of the number of multiplication and addition operations and the side information. Simulations are performed with quadrature phase shift keying modulation and a Saleh model power amplifier. The proposed scheme offers a significant PAPR reduction and bit error rate performance at approximately the same total complexity compared to the conventional partial transmit sequence and the enhanced partial transmit sequence (EPTS) techniques. The results show that at the same PAPR reduction, this scheme provides a complexity reduction of at least 42.3 $\%$ over that of the EPTS technique.
\end{abstract}

Keyword: $\quad$ BER; OFDM; PAPR; PTS; Gray code 